EPJ Web of Conferences 116, 08005 (2016)

DOI: $10.1051 /$ epjconf/201611608005

(C) Owned by the authors, published by EDP Sciences, 2016

\title{
Effect of atmospheric flux uncertainties on the determination of the neutrino mass hierarchy
}

\author{
Joakim Sandroos ${ }^{1, a}$, Thomas Erhardt ${ }^{1}$, Tim Arlen $^{2}$, and Sebastian Böser ${ }^{1}$ for the IceCube-Gen2 \\ Collaboration $^{\mathrm{b}}$ \\ ${ }^{1}$ Institute of Physics, University of Mainz, Staudinger Weg 7, 55099 Mainz, Germany \\ ${ }^{2}$ Dept. of Physics, Pennsylvania State University, University Park, PA 16802, USA
}

\begin{abstract}
The next generation of large-volume neutrino telescopes will include low-energy subarrays which will be able to measure neutrinos with energies of a few GeV. In this energy range the primary signal below the horizon is neutrinos created by cosmic ray interactions in the atmosphere. The measured event rate will depend on the neutrino mass hierarchy, allowing determination of this quantity to a significance level of about 3.5 sigma within a 5 -year period, mostly limited by systematic uncertainties. We present here the impact of the uncertainties on the atmospheric neutrino flux normalization on the determination of the neutrino mass hierarchy. We suggest constraining the systematic uncertainties by including the downgoing neutrino sample, which will increase the significance. This work was performed using simulation data from the low-energy extension to the IceCube detector located at the geographic south pole, PINGU, and is relevant to a wide range of other experiments.
\end{abstract}

\section{Introduction}

PINGU, the proposed low-energy extension to IceCube, located at the geographic south pole, will be able to measure neutrinos with an energy of a few GeV [1]. The experiment will be sensitive to the neutrino mass hierarchy $(\mathrm{NMH})$ due to neutrino oscillations enhanced by the MSW-effect of neutrinos traveling through the Earth. These kinds of measurements usually only include upgoing neutrinos, as the downgoing neutrinos will not have had time to oscillate and as such carry no information regarding the NMH. In this contribution we suggest including the downgoing neutrinos in order to constrain the uncertainties. The neutrino energies considered fall in the range of 1 to $80 \mathrm{GeV}$.

\section{Systematics}

The uncertainties usually considered in relation to PINGU analyses are: the ratios $v / \bar{v}, v_{e} / v_{\mu}$, the oscillation parameters $\theta_{13}, \theta_{12}, \Delta m_{31}^{2}$, and the effective area and the energy scale [1]. These however do

\footnotetext{
ae-mail: jsandroo@uni-mainz.de

${ }^{b}$ http://icecube.wisc.edu
}

This is an Open Access article distributed under the terms of the Creative Commons Attribution License 4.0, which permits unrestricted use, distribution, and reproduction in any medium, provided the original work is properly cited. 


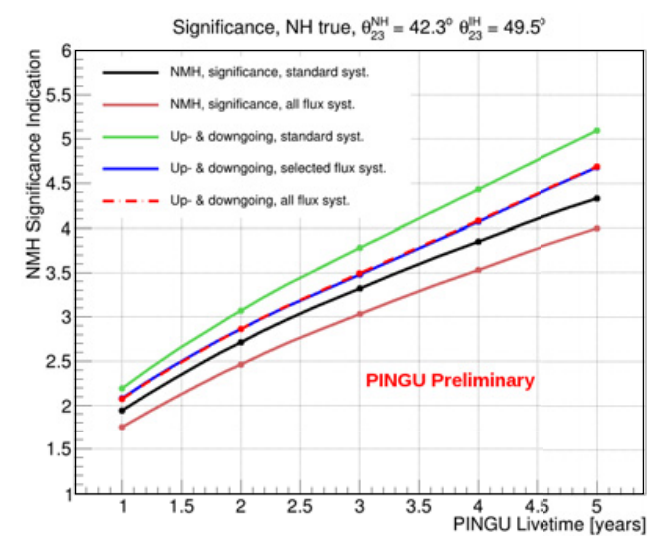

Figure 1. Significance vs. detector exposure for the neutrino mass hierarchy using PINGU. The black curve represents the standard set of 7 systematics, while the red includes the 5 most relevant atmospheric flux uncertainties. The green curve illustrates the significance when the downgoing neutrinos are included using the 7 standard systematics, while the red dashed curve includes the downgoing neutrinos and all systematics.

not touch upon the systematics stemming from the total normalization of the atmospheric neutrino flux. Using calculations from Barr et al. [2], we now incorporate the uncertainty on the atmospheric neutrino flux. This is considered from two sources: the primary cosmic ray flux and the hadronic interactions in the atmosphere. Barr et al. parametrizes the primary flux based on the GSHL model in the following way [2]:

$$
\Phi\left(E_{p}\right)=a\left[E_{p}+b \times \exp \left(c \sqrt{E_{p}}\right)\right]^{-d}
$$

where $\mathrm{d}$ is the atmospheric spectral index, $E_{p}$ is the energy per nucleon of the incoming cosmic ray, and $a, b$ and $c$ are normalization constants. The authors in [2] also consider the uncertainty in the hadronic interactions in the atmosphere, and divide the energy space into approximately independent regions as function of incoming cosmic ray energy and fraction of energy going into the decay products. The uncertainty of the atmospheric neutrino flux is thus different for each of these regions as well as for each of the four parameters on the primary CR flux, and are given individually in [2] as a function of energy $F_{i}(E)$. We take each function as a separate uncertainty input and associate each with a nuisance parameter $P_{i}$ to scale each function. Here the index $i$ refers to each of the independent uncertainties considered in [2] (Fig. 10). We can then vary the flux with the uncertainty as follows:

$$
\Phi(E) \rightarrow \Phi^{\prime}\left(E, P_{i}\right)=\Phi(E)\left[1+\sum_{i} P_{i} F_{i}(E)\right] .
$$

We use a Gaussian prior on the uncertainty parameters of $\pm 1 P_{i}$. We now get a contribution to the overall flux which is linear in the parameter $P_{i}$ by construction.

\subsection{Method}

The PINGU Simulation and Analysis software tool (PISA) is used for generating expected measurements from PINGU and analyzing those simulated data. It is not a traditional Monte Carlo generator: PISA takes as input external data such as a flux model and modifies it based on certain physical parameters to provide a model of the detector response under various assumptions of the 
nuisance parameters. In determining the neutrino mass hierarchy, a distinction between two possible physics scenarios is sought, and thus we compare simulated event rates in energy $\mathrm{E}$ and arrival direction $\cos \left(\theta_{z}\right)$ for each scenario and quantify their difference. This gives us the sensitivity to the NMH. Systematic uncertainties are implemented as individual parameters. In the following we investigate the impact of including the atmospheric neutrino flux uncertainties and the full sky on the neutrino mass hierarchy measurement. The flux is taken directly from MC flux tables in energy and $\cos \left(\theta_{z}\right)$, provided by Honda et al. [3] and is averaged over the azimuth angle. We use the flux at solar minimum. PISA incorporates several analysis methods; some based on a log likelihood ratio approach and other based on a $\Delta \chi^{2}$ approach under the Asimov assumption $[1,5,6]$. The $\Delta \chi^{2}$ method is computationally fast, but assumes a Gaussian likelihood space for all parameters. While this holds for the flux parameters, it is not true for all other parameters, especially the atmospheric mixing angle $\theta_{23}$. We perform the $\Delta \chi^{2}$ analysis for 10 values of $\theta_{23}$ over a suitable range around its $\Delta \chi^{2}$ minimum, and calculate the pulls for all other pa- rameters. Even if this will not find the smallest $\Delta \chi^{2}$ the method can be used to give a quick comparison between models with different systematic parameters. Some other parameters included are also non-linear to some extent, and while it is in principle possible to scan the parameter space, the computation time will grow geometrically with each parameter. The main point is that all our calculations contain the same error, which is not critical insofar as we are interested in the relative difference between our results. The impact calculated this way of including the flux systematics on the neutrino mass hierarchy is shown as the red curve in Fig. 1.

\section{Downgoing sample}

In the following we test the idea of also including the downgoing neutrino flux in the NMH analysis. Doing this, the uncertainty parameters can be constrained, since the oscillations have little to no impact on the downgoing flux, leaving only the systematics impact in that part of the parameter space. This in turn should lead to a rise in the significance. We make two assumptions:Because of the by IceCube veto around PINGU, we chose to ignore any additional background, and assume the same effective area for the downgoing flux as for the upgoing. These are both overly positive estimates and needs to be adjusted for future studies. We realize these are rough assumptions, however for this first feasability study they were assumed for speed reasons. we use the same methods of analysis as in the previous section. The results are included in Fig. 1, as the green and blue curves, where we see a clear boost in NMH significance compared to only the upgoing sample.

\subsection{Three samples}

We proceed to investigate the source of this significance increase, using three data samples: The pure upgoing region, the pure downgoing region and a combined region. The "pure" samples are simulated independently, while the combined sample is simulated over all angles of $\cos \left(\theta_{z}\right)$. Three effects which could additionally contribute to the significance are investigated: Oscillation in the downgoing region near the horizon, mis-reconstruction of events from one region to the other and the constraint on the uncertainties in the downgoing region. The NMH significance in the pure downgoing sample is found to be negligible as illustrated in Fig. 2. Using the three distinct samples it is possible to estimate the amount of contamination from mis-reconstructed events each pure region will experience from the other. This is done by comparing the pure samples to the corresponding regions of the combined sample respectively. The effect of each region on the other is found to be asymmetric significance wise: the upgoing region significance gets washed out, while the downgoing region significance gets boosted. Without including any uncertainties this effect is summarized in Table 1 . The effect of constraining the uncertainties is estimated by running our analysis twice: first using the pure downgoing sample, in order to get a constraint on the uncertainties - then again using those constraints as priors on the uncertainty 


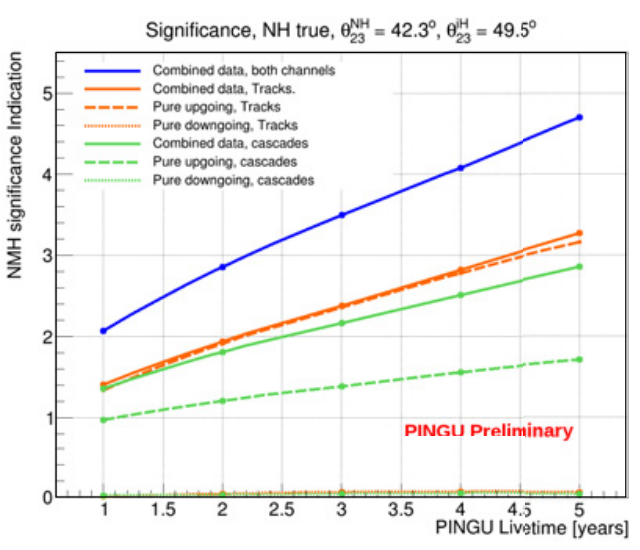

Figure 2. NMH Significance by sample and for track and cascade channels, as a function of time. The blue curve displays the combined sample and thus matches the blue curve in Fig. 1. The orange and green curves are from the track and cascade channels respectively. The dashed lines give the significance for the pure upgoing sample, while the fine dashed curves at the bottom represent the pure downgoing region.

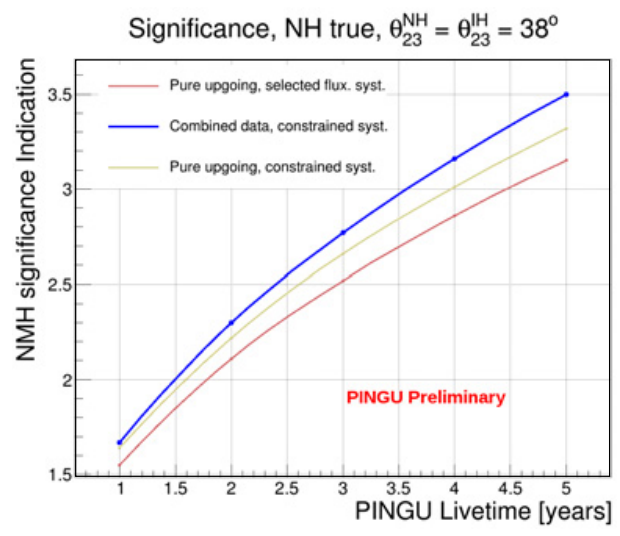

Figure 3. Contributions to the overall significance of the neutrino mass hierarchy as a function of time. The red curve is the significance in the pure upgoing sample when including the flux uncertainties. The yellow curve displays the significance in the pure upgoing sample when constraining the uncertainties based on the pure downgoing analysis. The blue curve is the total significance for the combined sample.

Table 1. NMH significance indication between samples for 5 years of livetime without uncertainties included.

\begin{tabular}{|c|c|c|} 
Singnificance Indication $\sqrt{\Delta \chi_{\min }^{2}}$ \\
\hline Sample & Tracks & Cascades \\
\hline \hline Pure Up & 7.67 & 9.11 \\
\hline Pure Down & 0.55 & 0.75 \\
\hline Comb. Up & 7.48 & 8.60 \\
\hline Comb. Down & 2.24 & 5.04 \\
\hline
\end{tabular}

parameters when analyzing the pure upgoing region. About half of the significance seem to come from upgoing events reconstructed as downgoing, while the other half seem to come from constraining the uncertainties. This is illustrated in Fig. 3. Note this is done at a mixing angle of $\theta_{23}=38^{\circ}$ for both hierarchies, so the overall significance is expected to be less than at the world average best-fit point. The red and blue curves in Fig. 3 have the same meaning as in Fig. 1, illustrating the pure upgoing data and the combined data respectively, while the yellow curve shows the pure upgoing sample with systematics constrained from the pure downgoing sample. We conclude the increase from the red to the yellow curve to be caused by better constraints on the uncertainties. The increase from the yellow to the blue curve stems from the oscillation signature carried above the horizon in mis-reconstructed events, which would otherwise have been lost. These two dominate over the negative effect of additional background in the up-going (signal) region.

\section{Closing remarks}

PINGU has started taking steps to quantify the impact of the systematic uncertainties of the atmospheric neutrino flux on the mass hierarchy measurement, and it turns out to be non-negligible. As a proof-ofconcept, we have studied the effect of using the downgoing neutrino flux as a reference, since we expect little to no NMH sensitivity in that region of our parameter space. This allowed us to obtain a tighter 
constraint on the flux systematics, which increases the sensitivity of the NMH measurement. A part of the improvement indeed stems from the tighter constraint on the uncertainties, but an equally strong effect is due to upgoing events being reconstructed as downgoing, which are lost if the downgoing region is not included. However we have assumed an identical effective area and a similar background for the downgoing region as in the upgoing region. Further studies are needed to address these shortcomings, but our analysis seem to indicate improvements in sensitivity to the $\mathrm{NMH}$ are possible by using the downgoing atmospheric neutrino flux.

\section{References}

[1] IceCube Gen2 collaboration, "PINGU Letter of Intent", arXiv: 1401.2046. Update in preparation.

[2] Barr et. al., "Uncertainties in Atmospheric Neutrino Fluxes", Phys. Rev. D74 (2006) 094009, arXiv: 0611266 [astro-ph]

[3] Honda, et al., "Atmospheric Neutrino Flux Calculation Using the NRLMSISE00 Atmospheric Model”, Phys. Rev. D75 (2007) 043006, arXiv: 1502 . 03916 [astro-ph.HE]

[4] R. A. Fisher, "On the mathematical foundations of theoretical statistics," Phil. Trans. Royal Soc. A220 (1922) $309\{368\}$

[5] L. Verde, "Statistical Methods in Cosmology", Lect. Notes Phys. 800, 147-177, 2010, arXiv: $0911.3105 v 1$ [astro-ph.CO]

[6] E. K. Akhmedov, S. Razzaque and A. Y. Smirnov. "Mass hierarchy, 2-3 mixing and CP-phase with Huge Atmospheric Neutrino Detectors", JHEP 1302 (2013), p. 08 